\title{
The surprising Far-UV spectrum of the polar BY Camelopardalis ${ }^{\star}$
}

\author{
M. Mouchet ${ }^{1,2}$, J.-M. Bonnet-Bidaud ${ }^{3}$, E. Roueff ${ }^{1}$, K. Beuermann ${ }^{4}$, D. de Martino ${ }^{5}$, J. M. Desert ${ }^{6}$, R. Ferlet ${ }^{6}$, \\ R. E. Fried ${ }^{7}$, B. T. Gänsicke ${ }^{8}$, S. B. Howell ${ }^{9}$, K. Mukai ${ }^{10}$, D. Porquet ${ }^{11}$, and P. Szkody ${ }^{12}$ \\ ${ }^{1}$ UMR 8102, associée au CNRS et à l'Université Paris 7, LUTH, Observatoire de Paris, Section de Meudon, \\ 92195 Meudon Cedex, France \\ 2 Université Paris 7 - Denis Diderot, 2 place Jussieu, 75005 Paris, France \\ 3 Service d'Astrophysique, DSM/DAPNIA/SAp, CE Saclay, 91191 Gif-sur-Yvette Cedex, France \\ ${ }^{4}$ Universtäts-Sternwarte Göttingen, Geismarlandstr. 11, 37083 Göttingen, Germany \\ 5 INAF, Osservatorio Astronomico di Capodimonte, Via Moiariello 16, 80131 Napoli, Italy \\ ${ }^{6}$ UMR 7095, associée au CNRS et à l'Université Paris 6, Institut d'Astrophysique de Paris, 98bis boulevard Arago, \\ 75014 Paris, France \\ 7 Braeside Observatory, Flagstaff, AZ 86002, USA \\ 8 Department of Physics and Astronomy, University of Southampton, Southampton SO17 1BJ, UK \\ 9 Astrophysics Group, Planetary Science Institute, Tucson, AZ 85705, USA \\ 10 NASA/Goddard Space Flight Center, Greenbelt, MD 20771, USA \\ 11 Max-Planck-Institut für extraterrestrische Physik, 85741 Garching, Germany \\ 12 Department of Astronomy, University of Washington, Seattle, WA 98195, USA
}

Received 2 October 2002 / Accepted 31 January 2003

\begin{abstract}
We report on the first far-UV observations of the asynchronous polar BY Cam made by the Far-Ultraviolet Spectroscopic Explorer (FUSE). The source is known to exhibit the most extreme NV/CIV emission resonance line ratio observed among polars. The FUSE observations reveal a OVI resonance line weaker than in the prototype of polars, AM Her, with the absence of a detectable narrow component. The OVI broad line is detected with an equivalent width of the same order as in AM Her, the blueward doublet component is clearly present but the redward component is strongly affected by $\mathrm{H}_{2}$ absorption. The presence of a strong NIII line and weak CIII lines also confirms the peculiar CNO line flux. We compare the resonance CNO line intensities with the predictions of the CLOUDY plasma code coupled to a geometrical model of the accretion column. Varying the temperature and/or intensity of the ionising spectrum is unable to reproduce the observed broad line ratios. A solution is obtained by significantly altering the element abundances with a strong depletion of $\mathrm{C}$, overabundance of $\mathrm{N}$ and a weak underabundance of $\mathrm{O}$. This confirms previous suggestions of non-solar abundances which may result from redistribution in the accreted material following nova outbursts and/or the secondary nuclear evolution. A very significant $\mathrm{H}_{2}$ absorption is observed in front of the source, a possible indication for either the existence of a dense interstellar cloud or of circumstellar material.
\end{abstract}

Key words. accretion, stars: individual: BY Cam - white dwarfs - cataclysmic variables - binaries: close - X-rays: H0538+608 - ultraviolet emission

\section{Introduction}

The hard X-ray source H0538+68/BY Cam was identified as a polar, a cataclysmic binary with a highly magnetized $(B \sim$ $10^{7}-10^{8} \mathrm{G}$ ) accreting white dwarf, after the detection of circularly polarized optical flux by Remillard et al. (1986). It was shown to exhibit a very peculiar UV emission line spectrum, with an emission resonance line ratio NV/CIV, more than ten times larger than what is usually observed in Polars

Send offprint requests to: M. Mouchet, e-mail: martine.mouchet@obspm.fr

$\star$ This work is based on data obtained from the NASA-CNES-CSA FUSE mission.
(Bonnet-Bidaud \& Mouchet 1987 (BM87)). It has been suggested that this unusual feature could be the result of a chemical evolution of the secondary, or of an unnoticed nova event leading to a CNO redistribution (BM87; Mouchet et al. 1990). The discovery that the source is slightly desynchronised (Silber et al. 1992) and the identification of a known nova, V1500 Cygni, as a polar which is also desynchronised (Schmidt \& Stockman 1991), gave further arguments to the nova hypothesis. However the picture has gradually changed as at least two synchronous polars, V1309 Ori (Szkody \& Silber 1996) and MN Hya (Schmidt \& Stockman 2001), have now been found to show a large NV/CIV ratio though less extreme than in BY Cam. The origin of these anomalous line strengths 
among polars is not clear. It might obviously result from nonsolar abundances in the accreted matter but could also be due to peculiar ionisation conditions. These effects may strongly affect our understanding of the evolution of these systems and their link to novae.

The FUSE satellite, operating in the range 905-1187 $\AA$ offers access to the OVI resonance line, a key for evaluating the $\mathrm{CNO}$ abundances. We report here on the first observation of BY Cam by this satellite. These observations were simultaneous with RXTE and EUVE observations as well as optical spectroscopy with the $3.5 \mathrm{~m}$ APO telescope and with the $9 \mathrm{~m} \mathrm{HET}$, and photometry at the $16^{\prime \prime}$ Braeside telescope whose results, including a temporal analysis, will be reported elsewhere.

\section{FUSE observations and reduction}

BY Cam was observed on 2000 January 10 and 16 by the FUSE satellite (Moos et al. 2000; Sahnow et al. 2000). The total wavelength range of 905-1187 $\AA$ is covered by means of four gratings and mirrors leading to bandpasses of about $200 \AA$, in such a way that the region below $\sim 1000 \AA$ is covered by two detectors, the central region by four and the region above $\sim 1100 \AA$ by two. During the first observation, the source was badly centered in the aperture and only the Jan. 16 data are presented below. The dataset consists of five exposures in the large aperture $\left(30^{\prime \prime} \times 30^{\prime \prime}\right)$, with exposure times ranging from 3.44 to $5.42 \mathrm{ks}$, resulting in a total $22 \mathrm{ks}$ on-source time. This covers two orbital cycles with only a small orbital phase interval $(\sim 0.12)$ not covered. The source was in the satellite continuous viewing zone and observations occurred mainly during "day" time, leading to the presence of strong geocoronal lines.

The data have been reduced using the Calfuse 1.7 .3 version of the standard pipeline. For each detector the five exposures were co-added with an eventual wavelength shift correction of one pixel. Velocity measurements have been corrected for the error in the heliocentric velocity value present in this calibration pipeline version. As the source is faint, the resulting signalto-noise ratio per resolution element is quite low (less than 3 ) so the original $(0.005 \AA)$ data have been binned into $0.1 \AA$ intervals. We report here on the mean phase-averaged spectrum.

\section{The average spectrum}

The average spectrum obtained by combining all detectors and exposures is shown in Fig. 1 where the strongest geocoronal lines have been removed for clarity. Some regions, such as around the NIV/HeII/H and HeII lines, are however possibly still contaminated by airglow and should be treated with care.

The continuum energy distribution is consistent with an extrapolation of the IUE high state spectrum. It can be described with a energy power law $F_{\lambda} \propto \lambda^{-1.43}$ if no reddening is assumed (-2.44 for $E_{B-V}=0.05$ (BM87)) with a total luminosity $L(910-1185 \AA)$ of $6.3 \times 10^{31} \mathrm{erg} \mathrm{s}^{-1}\left(12.2 \times 10^{31} \mathrm{erg} \mathrm{s}^{-1}\right.$ for $E_{B-V}=0.05$ ), assuming the source at $250 \mathrm{pc}$ (Gänsicke 1997) and a Cardelli extinction law (Cardelli et al. 1989) with a standard $A_{V} / E_{B-V}=3.1$ value. Broad emission lines are identified in Fig. 1, with intensities listed in Table 1. Pronounced

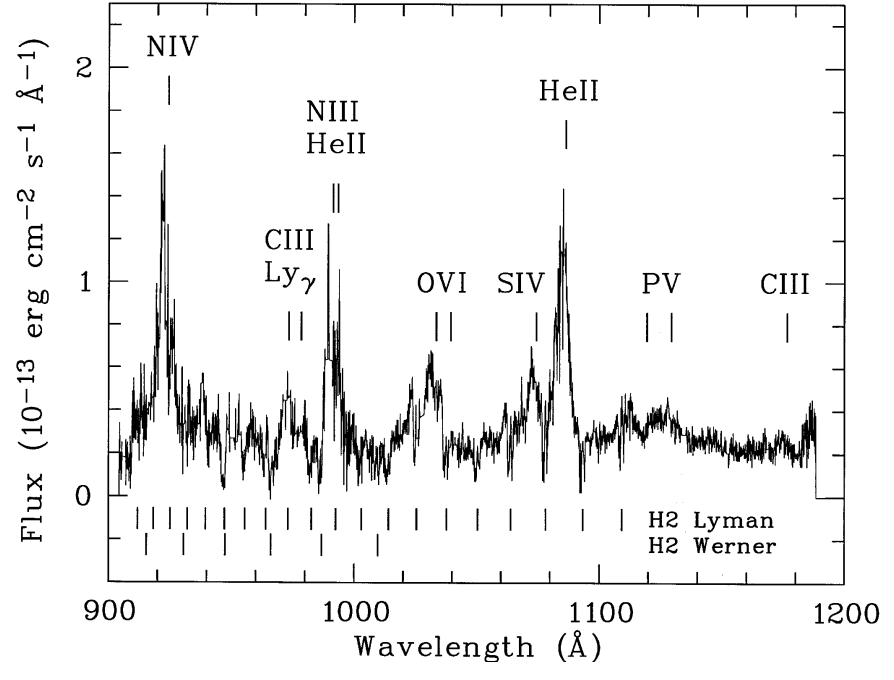

Fig. 1. The FUSE phase-averaged spectrum of BY Cam. The geocoronal lines have been removed for clarity and data are shown in $0.1 \AA$ bins. Remaining geocoronal lines can still contaminated the feature near $920 \AA$. The tick marks at the bottom indicate the positions of the $\mathrm{R}(0) \mathrm{H}_{2}$ absorption lines of the lowest vibrational level $v^{\prime \prime}=0$ for the Lyman and Werner bands.

Table 1. FUSE emission lines.

\begin{tabular}{lcll}
\hline \hline \multicolumn{1}{c}{ Line } & $\lambda(\AA)$ & $\operatorname{Flux}(1)$ & $E W(\AA)$ \\
\hline N IV/HeII/H & 923 & $4.8(+)$ & 14.5 \\
CIII & 977 & $0.6(+)$ & 2.7 \\
N III/ HeII & $989-92$ & 2.6 & 10.2 \\
O VI & $1032-38$ & 2.0 & 8.2 \\
S IV & 1073 & 0.8 & 2.1 \\
He II & 1085 & $4.1(+)$ & 11.0 \\
C III & 1175 & 0.1 & 0.6 \\
\hline
\end{tabular}

(1) Line flux in units of $10^{-13} \mathrm{erg} \mathrm{cm}^{-2} \mathrm{~s}^{-1}$.

Not corrected for reddening and/or $N_{\mathrm{H}_{2}}$.

(+) Possibly contaminated by airglow.

$\mathrm{H}_{2}$ molecular lines of the Lyman and Werner series are also clearly present (see Sect. 4.1).

Figure 2 shows an enlargement of the OVI line region. The OVI doublet in BY Cam is rather broad and weak, with the OVI $\lambda 1038$ component affected by strong $\mathrm{H}_{2}$ absorption. For comparison, the phase-averaged ORFEUS spectrum of AM Her (Mauche \& Raymond 1998), computed from retrieved archived data, is also shown in Fig. 2. Contrary to AM Her (see also Hutchings et al. 2002), no narrow OVI component is detected in BY Cam.

The OVI broad line intensities have been derived by fitting two Gaussians of same widths ( $F W H M$ of $1200 \mathrm{~km} \mathrm{~s}^{-1}$ ), at the doublet separation, convolved with an absorption with a $\mathrm{H}_{2}$ column density of $3 \times 10^{19}$ molecules $\mathrm{cm}^{-2}$ (cf. Sect. 4.1 ). The results are given in Table 2 and the best fit is shown in Fig. 2 (lower panel). Uncertainties in the doublet flux measurements have been computed by varying the amplitude of each Gaussian around the best value. They are given for the $99 \%$ confidence level, corresponding to a $\chi^{2}$ 


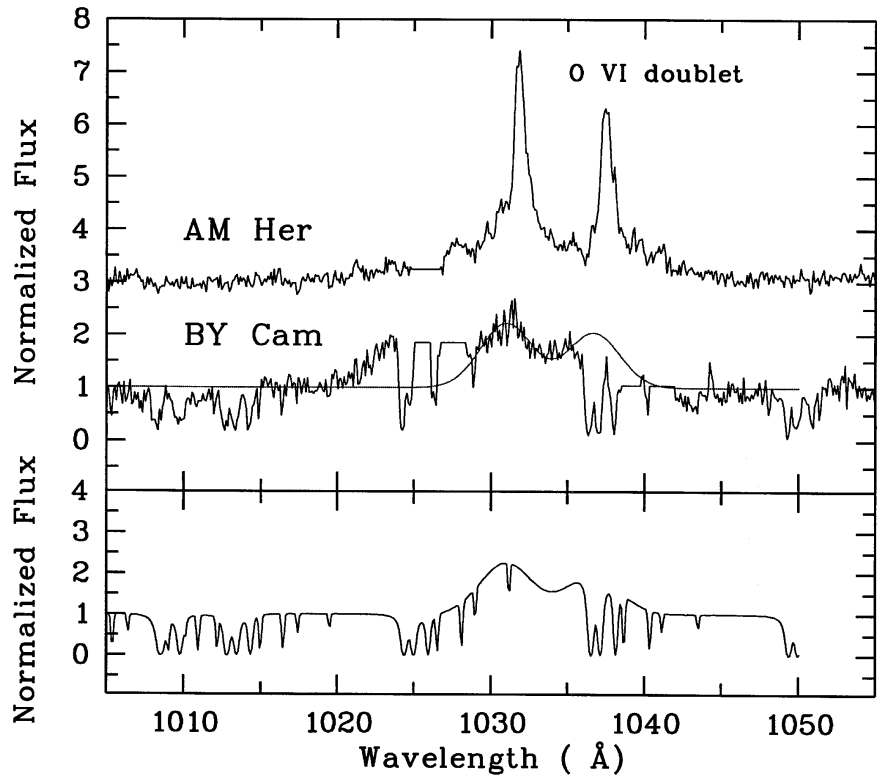

Fig. 2. Upper panel: the O VI doublet region as observed for AM Her (top, ORFEUS) and BY Cam (bottom, FUSE) with a best fit of two Gaussians superposed. The geocoronal lines, inluding $\operatorname{Ly} \beta$, are cut for clarity. Lower panel: the two Gaussian best fit including the $\mathrm{H}_{2}$ absorption (see text).

variation of 6.6 for a one parameter-fit. Line intensities corrected for $\mathrm{H}_{2}$ absorption and corresponding errors are $1.40_{-0.12}^{+0.13}$ and $1.09_{-0.17}^{+0.22} 10^{-13} \mathrm{erg} \mathrm{cm}^{-2} \mathrm{~s}^{-1}$, respectively for $\lambda 1032$ and $\lambda 1038$. Some caution should however be taken in interpreting the redward component intensity since it is affected by a strong $\mathrm{H}_{2}$ absorption and its red wing is also contaminated by an OI airglow contribution. For AM Her, the OVI total (broad + narrow) equivalent width is larger than for BY Cam but the equivalent width of the broad component alone is of the same order. The doublet intensity ratio of the broad component is also significantly different with a ratio of $\sim 1.3$ for BY Cam and 1.7 for AM Her. Assuming the source at $250 \mathrm{pc}$, the OVI broad line luminosity is $3.4 \times 10^{30} \mathrm{erg} \mathrm{s}^{-1}$, after correction for a $E_{B-V}=0.05$ interstellar reddening. This can be compared to the dereddened $\mathrm{NV}(\lambda 1240)$ and CIV $(\lambda 1549)$ luminosities of $1.7 \times 10^{31} \mathrm{erg} \mathrm{s}^{-1}$ and $2.9 \times 10^{30} \mathrm{erg} \mathrm{s}^{-1}$ respectively, derived from IUE observations (Zucker et al. 1995; Mouchet et al. 2002). The mean ratios $\mathrm{NV} / \mathrm{CIV}$ and OVI/CIV are 5.7 and 1.14 for BY Cam, while they are equal to 0.12 and 0.34 for AM Her. For this source, the values are derived from HUT spectra which wavelength range includes the three resonance lines (Greeley et al. 1999) and refer to the broad component only. Note however that for BY Cam the values obtained at different epochs are also affected by a mean intrinsic $\sim 35 \%$ variability (Mouchet et al. 1990; Zucker et al. 1995).

Contrary to AM Her, no strong narrow OVI line is observed in BY Cam, as shown in Fig. 2, where spectra for both sources are orbitally phase averaged. For BY Cam, the radial velocity amplitude of $\sim 250 \mathrm{~km} \mathrm{~s}^{-1}$ as derived for the optical narrow components (Mouchet et al. 1997), will introduce a broadening but will not be sufficient to smear out a narrow component $(F W H M \sim 0.5 \AA)$ as intense as in AM Her. The absence of
Table 2. Intensities and $E W$ of the OVI doublet.

\begin{tabular}{lrrrr}
\hline \hline & \multicolumn{2}{c}{ Narrow } & \multicolumn{2}{c}{ Broad } \\
& $\lambda 1032$ & $\lambda 1038$ & $\lambda 1032$ & $\lambda 1038$ \\
\hline BY Cam Flux (*) & $\leq 0.04$ & - & 1.4 & 1.1 \\
AM Her Flux $(*)$ & 6.6 & 5.9 & 12.8 & 7.7 \\
\hline BY Cam EW $(\AA)$ & $\leq 0.15$ & - & 5.2 & 4.4 \\
AM Her EW $(\AA)$ & 2.3 & 2.1 & 4.6 & 2.8 \\
\hline
\end{tabular}

(*) Line flux in units of $10^{-13} \mathrm{erg} \mathrm{cm}^{-2} \mathrm{~s}^{-1}$.

For AM Her: intensities derived from the mean ORFEUS spectrum (Mauche \& Raymond 1998).

For BY Cam: intensities derived assuming a $F W H M$ of $0.5 \AA$ (narrow) and $4 \AA$ (broad) and corrected for $\mathrm{H}_{2}$ molecular absorption.

narrow lines in BY Cam may be due to the absence of a strong soft X-ray component in BY Cam. However the polar VV Pup which is a soft X-ray source, does not either show obvious narrow components in the OVI lines (Hoard et al. 2002). Note that no conclusion can be drawn about the presence of a narrow component in the NV and CIV lines of BY Cam due to the lack of high resolution UV spectra.

\section{Discussion}

\subsection{The column density of molecular hydrogen}

Several $\mathrm{H}_{2}$ absorption lines of the Lyman and Werner electronic system bands are clearly detected in the average FUSE spectrum of BY Cam. The spectrum of molecular hydrogen was modelled assuming specific values of the total column density, a temperature of $80 \mathrm{~K}$ and a $b$ Doppler factor of $10 \mathrm{~km} \mathrm{~s}^{-1}$. The wavelengths, oscillator strengths and total radiative lifetimes of the upper levels of $\mathrm{H}_{2}$ transitions are given by Abgrall et al. (2000). The signal-to-noise ratio is too low to perform a fitting of the complete spectrum as done in FUSE studies towards translucent interstellar clouds (cf. Ferlet et al. 2000). We have compared the equivalent width measurements of the strongest $\mathrm{H}_{2}$ lines absorbed from the $J=0,1$, and 2 levels in the $v^{\prime}-0$ Lyman bands with $v^{\prime}=0$ to 4 , with those measured in the models. A best fit value is obtained for $\mathrm{a}_{2}$ column density $N_{\mathrm{H}_{2}}$ of $3 \times 10^{19}$ molecules $\mathrm{cm}^{-2}$. The model spectra for the Lyman $(0,0)$ and $(1,0)$ bands are displayed in Fig. 3 for 2 different values of the $\mathrm{H}_{2}$ column density of 1 and $3 \times 10^{19}$ molecules $\mathrm{cm}^{-2}$ together with the observed spectrum in the relevant spectral windows. The derived $\mathrm{H}_{2}$ column density of $3 \times 10^{19}$ molecules $\mathrm{cm}^{-2}$ should be correct within a factor of 2 . When using the $R(0)$ and $R(1)$ lines only, a model with a lower $b$ value of $3 \mathrm{~km} \mathrm{~s}^{-1}$ is marginally favoured. The presence of the $R(3)$ component at $1067.6 \AA$ and possibly at $1054.0 \AA$ seems to indicate a higher excitation temperature, but the quality of the spectra does not allow a reliable determination of its value. The individual spectra are also relatively noisy so that it is not possible to check reliably for possible changes with orbital phase.

From the absorption bump around $2200 \AA$, an upper limit of $E_{B-V} \leq 0.05$ has been derived for the source (BM87), which corresponds to a neutral $\mathrm{H}$ density $N_{\mathrm{H}_{1}} \leq 2.6 \times 10^{20} \mathrm{~cm}^{-2}$ (Shull \& van Steenberg 1985). This value is also consistent 


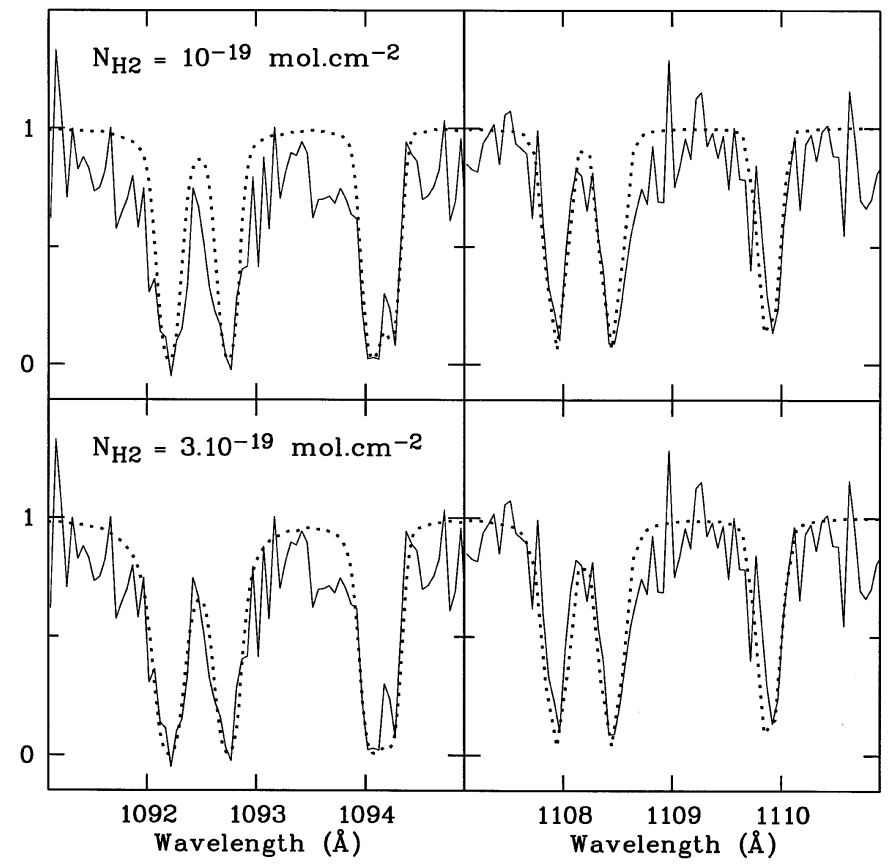

Fig. 3. Regions of the BY Cam normalized spectrum around the $\mathrm{H}_{2}$ absorption lines corresponding to the $0-0$ and $1-0$ vibrational transitions and models (dotted lines) for the best fit solution with $N_{\mathrm{H}_{2}}=$ $3 \times 10^{19} \mathrm{~mol} \mathrm{~cm}^{-2}$ (lower panels) and for $N_{\mathrm{H}_{2}}=1 \times 10^{19} \mathrm{~mol} \mathrm{~cm}^{-2}$ (upper panels) for illustration of the accuracy.

with the equivalent neutral $\mathrm{H}$ column density $N_{\mathrm{H}_{1}} \sim(1-2) \times$ $10^{20} \mathrm{~cm}^{-2}$ derived from the absorption cut-off in the X-ray spectra (Kallman et al. 1993, 1996). Taken at face value, this implies a very high $N_{\mathrm{H}_{2}} / N_{\mathrm{H}_{1}}$ ratio in the direction of the source. Assuming a standard interstellar medium, the expected $N_{\mathrm{H}_{2}}$ value, consistent with the observed reddening, is only in the range $5 \times 10^{11}-5 \times 10^{17} \mathrm{~cm}^{-2}$ (Savage et al. 1977). A large $N_{\mathrm{H}_{2}}$ value is unexpected for a relatively nearby source. It may result from an inhomogeneous interstellar medium with a molecular cloud in the line of sight but may also be linked with dense matter close to the source, possibly ejected during a prior nova event. Note that such $\mathrm{H}_{2}$ absorption has also being detected in the FUSE spectra of the supersoft binary QR And and has been suggested to come from a circumbinary location (Hutchings et al. 2001). The present FUSE absolute wavelength calibration is not accurate enough to derive precise values of the $\mathrm{H}_{2}$ line velocities and the systemic velocity of BY Cam is also not well known (in the range 1.3-141 $\mathrm{km} \mathrm{s}^{-1}$, Mason et al. 1989; Silber et al. 1992) so that no direct conclusion can yet be drawn about the possible circumbinary nature of the $\mathrm{H}_{2}$ cloud.

\subsection{Model of the OVI line and CNO abundances}

The broad resonance lines of the highly ionised species of CIV, NV, and OVI ( FWHM $\left.\sim 1200 \mathrm{~km} \mathrm{~s}^{-1}\right)$ are expected to be formed mainly in the accreting column, photoionised by the X-ray flux. Different attempts have been made to reproduce the line ratios using photoionisation models with a simplified geometry (cf. Mauche et al. 1997). We evaluate here the line luminosities computed using the CLOUDY plasma code (Ferland et al. 1998), coupled to a geometrical model of the accretion column. The ionising spectrum was defined as the sum of a $20 \mathrm{keV}$ bremsstrahlung, as observed in the RXTE observations, and of a $50 \mathrm{eV}$ blackbody with a bolometric luminosity of 0.1 times the hard X-ray luminosity (Ramsay et al. 1994). We have modelled the accretion column assuming a dipole geometry and free-fall velocities along the accretion column (cf. Stockman \& Schmidt 1996). The density then varies with the distance $r$ to the white dwarf, of radius $R_{\mathrm{wd}}$, as $n \sim n_{0}\left(r / R_{\mathrm{wd}}\right)^{-2.5}$ (Langer et al. 1982). We have set $n_{0}$, the density at the basis of the column, equal to $1.2 \times 10^{15} \mathrm{~cm}^{-3}$, corresponding to an accretion rate of $10^{16} \mathrm{~g} \mathrm{~s}^{-1}$, in agreement with the total X-ray luminosity of $6.6 \times 10^{32} \mathrm{erg} \mathrm{s}^{-1}$ for a distance of $250 \mathrm{pc}$ (Ramsay et al. 1994) and assuming a typical value of $10^{16} \mathrm{~cm}^{2}$ for the polar cap surface, and a $0.8 M_{\odot}$ white dwarf. The accretion column was approximated by a succession of different slabs of constant densities with a maximum extension chosen so that the density drops by a factor 4 from one slab to the next, up to a maximum extension of 50 times the white dwarf radius, i.e. about $1 / 4$ of the Roche lobe radius (Mouchet et al. 1997). The lateral extension of each region was assumed to follow the dipole geometry with a size varying as $\left(r / R_{\mathrm{wd}}\right)^{3 / 2}$. The illumination was approximated to be sideways which, taking into account of the bending of the column, is only true for the furthest parts of the column. We have verified however that, in all cases, the lowest slabs of higher densities do not contribute significantly to the total flux of the lines considered (Mouchet et al. 2002).

The luminosities of the three resonance lines $\operatorname{CIV}(\lambda 1549)$, $\mathrm{NV}(\lambda 1240)$ and $\mathrm{OVI}(\lambda 1035)$ were computed with this model, first assuming solar abundances. The results are shown in Fig. 4, where the $\mathrm{N} / \mathrm{C}$ and $\mathrm{O} / \mathrm{C}$ ratios are reported. Compared to the observed values, these ratios are significantly underestimated in this case. Changing the backbody temperature from $50 \mathrm{eV}$ to $10 \mathrm{eV}$ reduces significantly the $\mathrm{N}$ and $\mathrm{O}$ luminosities and does not improve the situation (see Fig. 4). However, a hidden very soft X-ray component, contributing significantly to the ionising flux, could still be present. For a neutral $\mathrm{H}$ column density of $2 \times 10^{20} \mathrm{~cm}^{-2}$, it is found that a $10 \mathrm{eV}$ blackbody component with a luminosity similar to that of the bremsstrahlung component, could remain undetected and still be compatible with the flux upper limit at $100 \AA$ of $6 \times 10^{-15} \mathrm{erg} \mathrm{cm}^{-2} \mathrm{~s}^{-1} \AA^{-1}$ derived from the EUVE observations. Though increasing significantly the $\mathrm{N}$ and $\mathrm{O}$ luminosities, it still falls short of the observed $\mathrm{N} / \mathrm{C}$ and $\mathrm{O} / \mathrm{C}$ ratios by factors 10 and 3 respectively. We therefore compute next the exact effect of varying the element abundances. An $\mathrm{N}$ abundance 10 times greater than the solar value was first considered. Since parts of the column are optically thick, the N line luminosity is not increased proportionally but only by a factor $\sim 4$, with a corresponding small decrease in the $\mathrm{C}$ and $\mathrm{O}$ lines but not sufficient to reproduce the observed values (see Fig. 4). Assuming our adopted model to be valid, the observed line ratios require highly non-solar abundances. As the BY Cam line luminosities are somewhat variable, we did not attempt here to obtain an exact fit in the abundances but we show in Fig. 4 a combination where $\mathrm{C}, \mathrm{N}$ and $\mathrm{O}$ are respectively lower by a factor 25 , higher by 8 and lower by 2 , compared to solar 


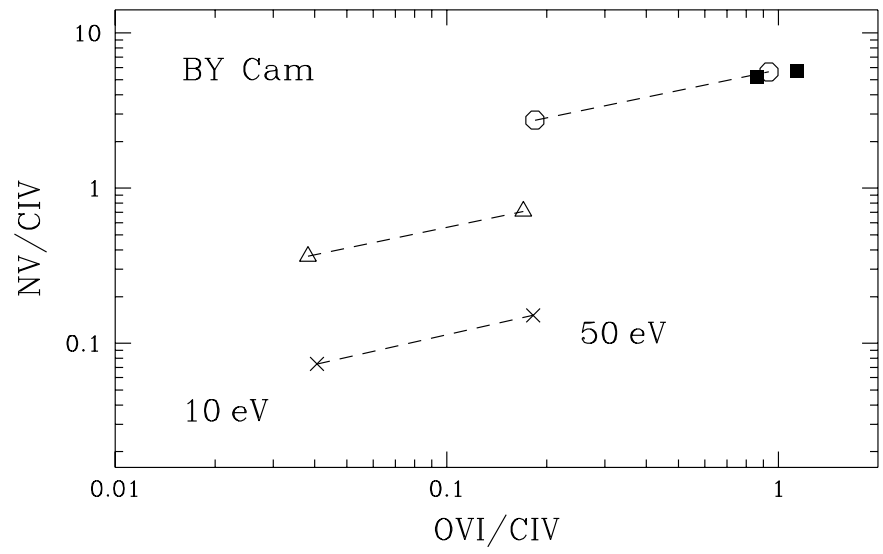

Fig. 4. Predicted broad line ratios computed from CLOUDY plasma code with a simplified model of the accretion column (see text). Values for different ionising spectra with a $20 \mathrm{keV}$ bremsstrahlung and a blackbody temperature ranging from $10 \mathrm{eV}$ to $50 \mathrm{eV}$ are shown (dashed line) compared to the BY Cam observed value (filled squares; left: with no reddening correction, right: after reddening correction). Ratios are plotted for solar abundances (crosses). Effects of varying abundances are also shown with a 10 times solar value for $\mathrm{N}$ (open triangles) and a combination where $\mathrm{C}, \mathrm{N}$ and $\mathrm{O}$ are respectively lower by a factor 25 , higher by 8 and lower by 2 compared to solar values (open rounds) which fits best the observed value.

values. With these non-solar abundances, the observed dereddened ratios are well reproduced, within a factor 1.01 and 1.22 , respectively for NV/CIV and OVI/CIV. Other combinations may of course be valid and a more detailed modelling will be presented elsewhere (Bonnet-Bidaud et al. 2003, in preparation). These first precise computations of the abundance effects confirm our previous suggestions (BM87, Mouchet et al. 1990) that the peculiar line intensities in BY Cam may be due to a conjugated overabundance of $\mathrm{N}$ with a underabundance of C. The strong line of NIII (990 $\AA$ ) observed here (as NIV $(1718 \AA))$, together with the weaknesses of the CIII lines at $977 \AA$ and at $1175 \AA$, confirm this. The oxygen abundance deviates only slightly from the solar value.

\subsection{Origin of the peculiar CNO composition}

The explanation of such highly non-solar values for some elements is still pending. The nova hypothesis is tempting as the by-product of the $\mathrm{H}$-burning $\mathrm{CNO}$ cycle is a ${ }^{14} \mathrm{~N}$ production at the expense of ${ }^{12} \mathrm{C}$, leading to a $\mathrm{CN}$ redistribution. But the recent nova V1500 Cyg does not show abnormal UV line intensities (Schmidt et al. 1995), and the ejecta observed in a large sample of novae show enrichment in all three C, N, O elements (Livio \& Truran 1994), indicating dredge-up of matter from the underlying CO core (Starrfield et al. 2000). However multicycle models lead to a rearrangement of CNO abundances by reprocessing a large quantity of carbon and some fraction of oxygen into nitrogen (Kovetz \& Prialnik 1997). Enhancement of $\mathrm{N}$ and depletion of $\mathrm{C}$ can indeed be obtained in the context of evolutionary models of semi-detached systems in which the secondary surface is polluted by re-accretion of material ejected during nova outburts (Marks \& Sarna 1998). But the enrichment by a nova-type explosion requires efficient reaccretion of the burnt material as pointed out by Stehle \& Ritter (1999).

As about $1 / 3$ of cataclysmic variables might descend from supersoft X-ray binaries (Schenker et al. 2002), the required $\mathrm{CNO}$ composition in $\mathrm{BY}$ Cam can result from steady nuclear burning phases which occur during this evolutionary stage. Interestingly, one of these sources, XMMU J004319.4+411758, might be a progenitor of a magnetic cataclysmic variable (King et al. 2002). We note that weak thermonuclear runaways can also have occurred in dwarf novae such as U Gem for which the white dwarf spectrum reveals a depletion of carbon (Sion et al. 1998) or such as BZ UMa and EY Cyg whose UV spectra exhibit a strong NV line and a weak CIV line (Sion 2002; Szkody 2002).

Another possibility for the origin of the peculiar composition requires an evolved secondary whose external layers have been stripped off during episodes of mass loss. Computed evolutionary paths of binary systems can produce partially nuclear evolved secondaries prior to contact (Howell 2001), if the donor star is sufficiently massive (Haswell et al. 2002). This might explain the pathological CNO UV line ratios observed in the long period system AE Aqr (Mouchet et al. 2002), as has been demonstrated by Schenker et al. (2002), but evolutionary models adapted for the orbital parameters of BY Cam have still to be computed.

Stronger constraints on the abundance values should be obtained from the $\mathrm{H}$-like and He-like lines of $\mathrm{C}, \mathrm{N}$, and $\mathrm{O}$ which are accessible with XMM-Newton and Chandra. Indeed the first XMM-Newton RGS spectra of BY Cam show emission lines tentatively identified with NVI and NVII lines (Ramsay \& Cropper 2002). Other hydrogen-like and helium-like lines of $\mathrm{C}$ and $\mathrm{O}$ are expected in the RGS range. Longer exposed spectra are needed to achieve a detailed model of the X-ray emitted plasma that would provide a good evaluation of the $\mathrm{CNO}$ abundance.

Acknowledgements. We are very grateful to Alain Lecavelier des Étangs for his helpful support in FUSE observing preparation and data reduction.

\section{References}

Abgrall, H., Roueff, E., \& Drira, I. 2000, A\&AS, 141, 297

Bonnet-Bidaud, J. M., \& Mouchet, M. 1987, A\&A, 188, 89

Cardelli, J. A., Clayton, G. C., \& Mathis, J. S. 1989, ApJ, 345, 245

Ferland, G. J., Korista, K. T., Verner, D. A., et al. 1998, PASP, 110, 761

Ferlet, R., André, M., Hébrard, G., et al. 2000, ApJ, 538, L69

Gänsicke, B. T. 1997, Ph.D. Thesis, Universität Göttingen

Greeley, B. W., Blair, W. P., Long, K. S., \& Raymond, J. C. 1999, ApJ, 513,491

Haswell, C. A., Hynes, R. I., King, A. R., \& Schenker, K. 2002, MNRAS, 332, 928

Hoard, D. W., Szkody, P., Ishioka, R., et al. 2002, AJ, 124, 2238

Howell, S. B. 2001, PASJ, 53, 675

Hutchings, J. B., Crampton, D., Cowley, A. P., Schmidtke, P. C., \& Fulleton, A. W. 2001, AJ, 122, 1572

Hutchings, J. B., Fulleton, A. W., Cowley, A. P., \& Schmidtke, P. C. 2002, AJ, 123, 2841 
Kallman, T. R., Schlegel, E. M., Serlemitsos, P. J., et al. 1993, ApJ, 411,869

Kallman, T. R., Mukai, K., Schlegel, E. M., et al. 1996, ApJ, 466, 973

King, A. R., Osborne, J. P., \& Schenker, K. 2002, MNRAS, 329, L43

Kovetz, A., \& Prialnik, D. 1997, ApJ, 477, 356

Langer, S. H., Chanmugam, G., \& Shaviv, G. 1982, ApJ, 258, 289

Livio, M., \& Truran, J. W. 1994, ApJ, 425, 797

Marks, P. B., \& Sarna, M. J. 1998, MNRAS, 301, 699

Mason, P. A., Ramsay, G., Andronov, I., et al. 1989, MNRAS, 295, 511

Mauche, C. W., \& Raymond, J. C. 1998, ApJ, 505, 869

Mauche, C. W., Lee, Y. P., \& Kallman, T. R. 1997, ApJ, 477, 832

Moos, H. W., Cash, W. C., Cowie, L. L., et al. 2000, ApJ, 538, L1

Mouchet, M., Bonnet-Bidaud, J. M., \& Hameury, J. M. 1990a, in Accretion-Powered Compact Binaries, ed. C. Mauche (CUP), 247

Mouchet, M., Bonnet-Bidaud, J. M., Hameury, J. M., \& Acker, A. 1990b, ESA SP-310, 423

Mouchet, M., Bonnet-Bidaud, J. M., Somov, N. N., \& Somova, T. A. 1997, A\&A, 324, 109

Mouchet, M., Bonnet-Bidaud, J. M., Abada-Simon, M., et al. 2002, in Classical Nova Explosions, ed. M. Hernanz, \& J. José, AIP, 67

Ramsay, G., \& Cropper, M. 2002, MNRAS, 334, 805

Ramsay, G., Mason, K., Cropper, M., et al. 1994, MNRAS, 270, 692
Remillard, R. A., Bradt, H. V., McClintock, J. E., et al. 1986, ApJ, 302, L11

Sahnow, D. J., Moos, H. W., Ake, T. B., et al. 2000, ApJ, 555, 839

Savage, B. D., Drake, J. F., Budich, W., \& Bohlin, R. C. 1977, ApJ, 216, 291

Schenker, K., King, A. R., Kolb, U., Wynn, G. A., \& Zhang, Z. 2002, MNRAS, 337, 1105

Schmidt, G. D., \& Stockman, H. S. 1991, ApJ, 371, 749

Schmidt, G. D., \& Stockman, H. S. 2001, ApJ, 548, 410

Schmidt, G. D., Liebert, J., \& Stockman, H. S. 1995, ApJ, 441, 414

Shull, J. M., \& van Steenberg, M. E. 1985, ApJ, 294, 599

Silber, A., Bradt, H. V., Ishida, M., et al. 1992, ApJ, 389, 704

Sion, E. M., Cheng, F. H., Szkody, P., et al. 1998, ApJ, 496, 449

Sion, E. M. 2002, in Classical Nova Explosions, ed. M. Hernanz, \& J. José, AIP, 21

Starrfield, S., Truran, J. W., \& Sparks, W. M. 2000, New Ast. Rev., 44, 81

Stehle, R., \& Ritter, H. 1999, MNRAS, 309, 245

Stockman, H. S., \& Schmidt, G. D. 1996, ApJ, 468, 883

Szkody, P. 2002, in Classical Nova Explosions, ed. M. Hernanz, \& J. José, AIP, 28

Szkody, P., \& Silber, A. 1996, AJ, 112, 239

Zucker, D. B., Raymond, J. C., Silber, A., et al. 1995, ApJ, 449, 310 